\title{
Forced Convective Cooling via Acoustic Streaming in a Narrow Channel Established by a Vibrating Piezoelectric Bimorph
}

\author{
QUN WAN, TAO WU, JOHN CHASTAIN, WILLIAM L. ROBERTS, \\ ANDREY V. KUZNETSOV and PAUL I. RO \\ Department of Mechanical and Aerospace Engineering, North Carolina State University, Raleigh, \\ NC27695-7910,U.S.A., E-mail: avkuznet@eos.ncsu.edu
}

Received 8 January 2005; accepted 8 February 2005

\begin{abstract}
Forced convection in a narrow channel is investigated both numerically and experimentally. The flow field is established through the mechanism of acoustic streaming. This is accomplished by high frequency vibration of one of the channel walls, which is composed of a piezoelectric bimorph. In the numerical computations, the Navier-Stokes equations are decomposed into the acoustic equations and the streaming equations by the perturbation method. The acoustic field is first numerically obtained, which provides the driving force for the streaming field. The streaming field and the associated temperature field are then obtained numerically. Heat losses from a heat source are measured to determine the efficiency of this as a cooling method. The air-flow patterns in the channel between the heat source and the bimorph actuator are visualized using the particle tracking velocimetry. The visualization clearly shows that vortical streaming (acoustic streaming) can be induced by bimorph vibration, which enhances heat transfer between the heat source and the surrounding air. The temperature decreases obtained computationally and experimentally are in good agreement.
\end{abstract}

Key words: acoustic streaming, forced convection, cooling enhancement, vibrating surface

\section{Introduction and Theory}

The acoustic field in a fluid with attenuation, due to viscosity and thermal conduction, is always accompanied by the unidirectional flow called acoustic streaming. Bradley [1] and Nyborg [2] analyzed this phenomenon by assuming that the acoustic streaming does not affect the first order acoustic field and appears in second order terms. Utilizing the perturbation method and the compressible Navier-Stokes equations, the first order acoustic equations and second order acoustic streaming equations are obtained. The acoustic field with attenuation is governed by the following first order acoustic equations:

$$
\frac{\partial \rho_{1}}{\partial t}+\rho_{0} \nabla \cdot \mathbf{u}_{1}=0
$$




$$
\begin{aligned}
& \nabla \nabla \cdot \mathbf{u}_{1}+\frac{\mu}{\rho_{0} c_{0}^{2}} \nabla^{2} \frac{\partial \mathbf{u}_{1}}{\partial t}+\frac{\mu_{B}+\mu / 3}{\rho_{0} c_{0}^{2}} \nabla \nabla \cdot \frac{\partial \mathbf{u}_{1}}{\partial t}-\frac{1}{c_{0}^{2}} \frac{\partial^{2} \mathbf{u}_{1}}{\partial t^{2}}=0 \\
& p_{1}=c_{0}^{2} \rho_{1}
\end{aligned}
$$

where $\mathbf{u}_{1}=\left(u_{1}, v_{1}\right)$ is the acoustic velocity vector; $\rho_{1}$ and $p_{1}$ are the acoustic density and acoustic pressure, respectively; $\rho_{0}$ is the density of the fluid at rest; $c_{0}$ is the speed of sound; $t$ is the time; $\nabla$ is the differential operator in coordinates $(x, y)$; and $\mu$ and $\mu_{B}$ are the fluid dynamic viscosity and bulk viscosity, respectively.

The streaming field is governed by the following second order acoustic streaming equations:

$$
\begin{array}{r}
\rho_{0} \nabla \cdot \mathbf{u}_{\mathrm{dc}}=\dot{m} \\
-\mu \nabla^{2} \mathbf{u}_{\mathrm{dc}}-\left(\mu_{B}+\mu / 3\right) \nabla \nabla \cdot \mathbf{u}_{\mathrm{dc}}+\nabla p_{\mathrm{dc}}=\mathbf{f}_{b}
\end{array}
$$

where $\mathbf{u}_{d c}=\left(u_{\mathrm{dc}}, v_{\mathrm{dc}}\right)$ is the streaming velocity vector and $p_{\mathrm{dc}}$ is the streaming pressure. The acoustic field drives the streaming field through the acoustic intensity $\mathbf{I}=$ $p_{1} \mathbf{u}_{1}\left(\dot{m}=-\frac{1}{c_{0}^{2}} \nabla \cdot\langle\mathbf{I}\rangle\right)$ and the driving force $\mathbf{f}_{b}=-\frac{1}{c_{0}^{2}}\left\langle p_{1} \frac{\partial \mathbf{u}_{1}}{\partial t}\right\rangle-\rho_{0}\left\langle\left(\mathbf{u}_{1} \cdot \nabla\right) \mathbf{u}_{1}\right\rangle$, where \langle\rangle denotes time average of a variable.

In this paper, the cooling efficiency of the acoustic streaming within a narrow channel is investigated. As shown in the schematic diagram in Figure 1, the channel is composed of a bimorph beam, which is fixed at both ends and thus vibrates in its first standing wave mode at certain cross section electric field intensity, and a stationary beam, which is attached to a rectangular solid heat source outside of the channel. The heat source has a constant heat generation rate and is insulated on all sides except the side attached to the stationary beam, allowing heat to be dissipated through the channel only. The bimorph beam is kept at a constant temperature, and the thickness of both beams is negligible. The coordinate setup for this problem is also shown in Figure 1. The displacement $\mathbf{d}(t, x)$ of the bimorph beam is described as:

$$
\mathbf{d}(t, x)=\mathbf{j} A \sin \omega t \sin k x, \quad \text { when } x \text { within the vibration region }
$$

where $A, \omega$ and $k$ are the displacement amplitude, angular frequency and the wave number of the acoustic field, respectively, and $\mathbf{j}$ is the unit vector along $y$ direc-

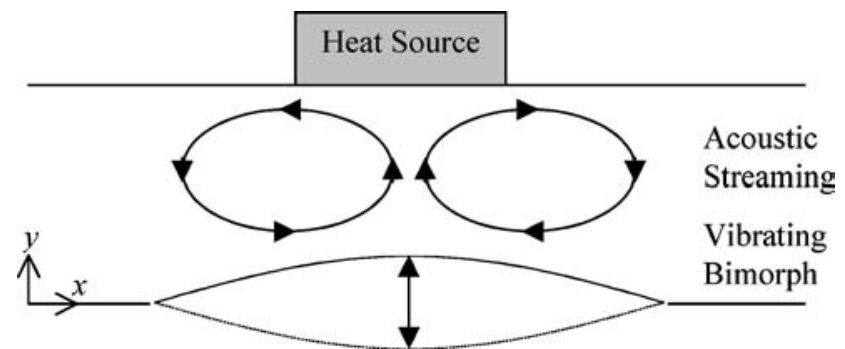

Figure 1. Schematic diagram of the cooling device based on acoustic streaming. 
tion. According to Wan and Kuznetsov [3], derivation of the beam displacement Equation (6) gives the beam vibration velocity, which by Taylor expansion, is the summation of acoustic velocity at the mean position of the vibrating beam

$$
\mathbf{u}_{1}(t, x, 0)=\mathbf{j} \omega A \cos \omega t \sin k x \text {, when } x \text { is within the vibration region }
$$

and the acoustic streaming velocity at the mean position of the vibrating beam

$$
\mathbf{u}_{\mathrm{dc}}(x, 0)=\left\langle(\mathbf{d} \cdot \nabla) \mathbf{u}_{1}(t, x, 0)\right\rangle \text {, when } x \text { is within the vibration region }
$$

where higher order components are neglected.

Instead of solving the time-dependent wave Equations (1)-(3), this paper utilizes the reduced form of these equations derived in Wan and Kuznetsov [3], which in the steady state and complex number form can be presented as

$$
\begin{aligned}
& {\left[1+i \omega\left(A_{\mu}+B_{\mu}\right)\right] \frac{\partial^{2} \hat{u}}{\partial x^{2}}+i \omega A_{\mu} \frac{\partial^{2} \hat{u}}{\partial y^{2}}+\left[1+i \omega B_{\mu}\right] \frac{\partial^{2} \hat{v}}{\partial x \partial y}+\frac{\omega^{2}}{c_{0}^{2}} \hat{u}=0} \\
& i \omega A_{\mu} \frac{\partial^{2} \hat{v}}{\partial x^{2}}+\left[1+i \omega\left(A_{\mu}+B_{\mu}\right)\right] \frac{\partial^{2} \hat{v}}{\partial y^{2}}+\left[1+i \omega B_{\mu}\right] \frac{\partial^{2} \hat{u}}{\partial x \partial y}+\frac{\omega^{2}}{c_{0}^{2}} \hat{v}=0 \\
& \hat{p}=i \frac{c_{0}^{2} \rho_{0}}{\omega}\left(\frac{\partial \hat{u}}{\partial x}+\frac{\partial \hat{v}}{\partial y}\right)
\end{aligned}
$$

where $\hat{\mathbf{u}}=(\hat{u}, \hat{v})$ is the amplitude of the vibrating acoustic velocity, $\hat{p}$ is the amplitude of the vibrating pressure, and $i$ is the imaginary unit. The attenuation related parameters are $A_{\mu}=\frac{\mu}{\rho_{0} c_{0}^{2}}$ and $B_{\mu}=\frac{\mu_{B}+\mu / 3}{\rho_{0} c_{0}^{2}}$, respectively. According to Wan and Kuznetsov [3], the driving terms in Equations (4) and (5) can be represented in the acoustic amplitudes as

$$
\dot{m}=-\frac{1}{2 c_{0}^{2}} \operatorname{Re}[\nabla \cdot(\overline{\hat{p}} \hat{\mathbf{u}})]
$$

and

$$
\mathbf{f}_{b}=-\frac{1}{2 c_{0}^{2}} \operatorname{Re}\left[i \overline{\hat{p}} \hat{\mathbf{u}}+\rho_{0}(\overline{\hat{\mathbf{u}}} \cdot \nabla) \hat{\mathbf{u}}\right]
$$

where $\operatorname{Re}(\hat{a})$ represents the real part of the variable $\hat{a}$ and the overbar denotes the conjugate of the complex variable.

Accordingly, the amplitude of the acoustic velocity due to this beam vibrating at the mean position can be derived from Equation (7) as

$$
\hat{u}(x, 0)=0, \hat{v}(x, 0)=\omega A \sin k x \text {, when } x \text { is within the vibration region. }
$$

The acoustic streaming velocity at the mean position of the vibrating beam, Equation (8), is rewritten as

$$
\mathbf{u}_{\mathrm{dc}}(x, 0)=\frac{1}{2} A \sin k x \operatorname{Re}[\nabla \cdot(i \hat{\mathbf{u}})], \text { when } x \text { is within the vibration region. }
$$


Since the vibrating acoustic field is assumed to be adiabatic (generally a good assumption), the accompanying unidirectional streaming flow contributes to the convective heat transfer and can carry away the heat from the heated surface. According to Wan and Kuznetsov [3], the steady-state energy equation for the streaming flow is

$$
\left(\mathbf{u}_{\mathrm{dc}} \cdot \nabla\right) \theta=a \nabla^{2} \theta
$$

where $\theta(x, y)=T(x, y)-T_{w}$ is the difference between the temperature $T(x, y)$ and reference temperature $T_{w}$, and $a$ is the thermal diffusivity of the fluid.

In this analysis, the heat generation rate is constant and the steady-state energy equation can be used, which for the heat source can be written as:

$$
k_{c} \nabla^{2} \theta+\dot{q}=0
$$

where $k_{c}$ is the thermal conductivity of the solid and $\dot{q}$ is the constant heat generation rate within the heat source.

\section{Numerical Procedure}

To simulate the experimental results obtained on the cooling efficiency of the acoustic streaming, the acoustic field within the gap, which is governed by Equations (9)(11) and driven by the acoustic source (Equation (13)) on the vibrating beam, is first obtained numerically. Then, the driving terms given by Equations (12a,b) are computed and the streaming field governed by Equations (4) and (5) together with the boundary condition (14) is also obtained numerically. Consequently, the convection temperature field of the acoustic streaming, which is governed by Equation (15) and coupled with the heat source conduction field governed by Equation (16) at the stationary beam, is obtained numerically. The cooling efficiency is investigated by comparing the mean temperatures of the heat source surface when the acoustic streaming is on and off. The temperature field with acoustic streaming off is obtained by simply setting the acoustic streaming velocity, $\mathbf{u}_{\mathrm{dc}}$, to zero; in this case heat conduction is the only cooling mechanism.

The boundary conditions at the open ends of the channel for all the governing equations are zero-gradient boundary conditions except for the first order acoustic Equations (9)-(11), where the second order non-reflective boundary conditions are applied (Givoli [4], Wan and Kuznetsov [3, 5]). On the surface of the stationary beam and the fixed part of the vibrating beam, no-slip boundary conditions and zero pressure gradients in the normal directions are utilized. Adiabatic boundary conditions are utilized on all sides of the heat source except for the interface with the stationary beam, where the temperature and heat flux at the heat source side couple with those of the fluid side in the narrow channel.

All governing equations are discretized by a finite volume method and numerically solved by the Gauss-Seidel iteration method. In addition, the SIMPLER 


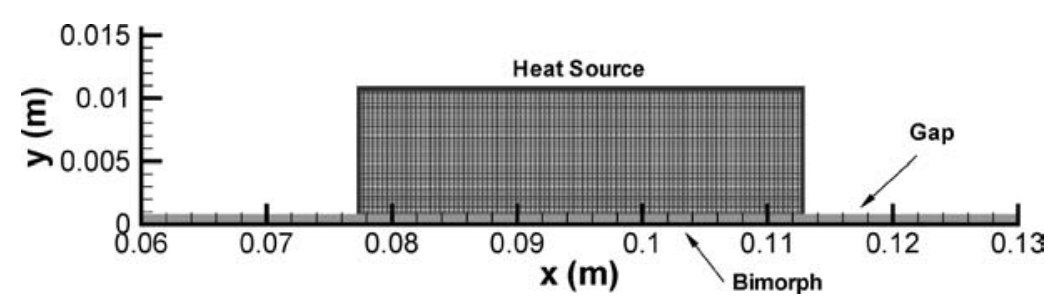

Figure 2. Computational meshes for heat source and the gap.

method is utilized in solving the acoustic streaming Equations (4) and (5). A uniform $501 \times 21$ mesh is generated in the channel domain and a uniform $94 \times 51$ mesh is generated in the heat source domain. Both meshes are shown in Figure 2. The parameters from the experiments are: $280 \mathrm{~Hz}$ vibrating frequency, $130 \mu \mathrm{m}$ vibrating amplitude, $1 \mathrm{st}$ mode resonance, $0.0635 \mathrm{~m}$ length of vibrating region, $0.03556 \mathrm{~m} \times 0.0254 \mathrm{~m} \times 0.01016 \mathrm{~m}$ silicon heat source with $3.9 \mathrm{~W}$ power, $650 \mu \mathrm{m}$ gap filled with dry air. In computations, the fixed regions near both ends of the bimorph are set to be $0.0635 \mathrm{~m}$. Since the numerical model is two-dimensional, the heat generation rate is calculated based on the power and the dimensions of the heat source.

\section{Visualization of Flow Patterns}

\subsection{EXPERIMENTAL APPARATUS AND DESIGN}

Visualization of the induced flow field between the heat source and bimorph actuator was performed using Particle Tracking Velocimetry (PTV). The experimental apparatus for flow visualization is shown in Figure 3. The piezoelectric bimorph beam was mounted on a stand and held rigidly on all four corners. The heat source was then mounted above the bimorph, with an adjustable gap between them. The air gap between the heat source and bimorph in the experiments was controlled by four micrometer heads, and varied between 1 and $3 \mathrm{~mm}$. The piezoelectric bimorph was excited at the beam's fundamental resonance frequency by supplying a sinusoidal signal from a function generator. The amplitude was $50 \mathrm{~V}$. No power was applied to the stationary beam for flow visualization.

To obtain flow field patterns, the channel gap was seeded with 1-micron diameter aluminum oxide particles. After allowing sufficient time for the bulk motion induced by the seed particle injection to dampen out, the particles were imaged at a magnification of 1:1 using a macro lens. A laser sheet was formed from Argon ion laser operating at $514 \mathrm{~nm}$ and directed down the length of the gap. Because of the high aspect ratio of the gap, a long focal length lens was used to form the sheet, giving a nominal thickness of 400 microns. The laser sheet did not propagate down the centerline of the gap, but was moved towards the camera such that it was one quarter of the width from the camera edge. This allowed a slightly larger field of 


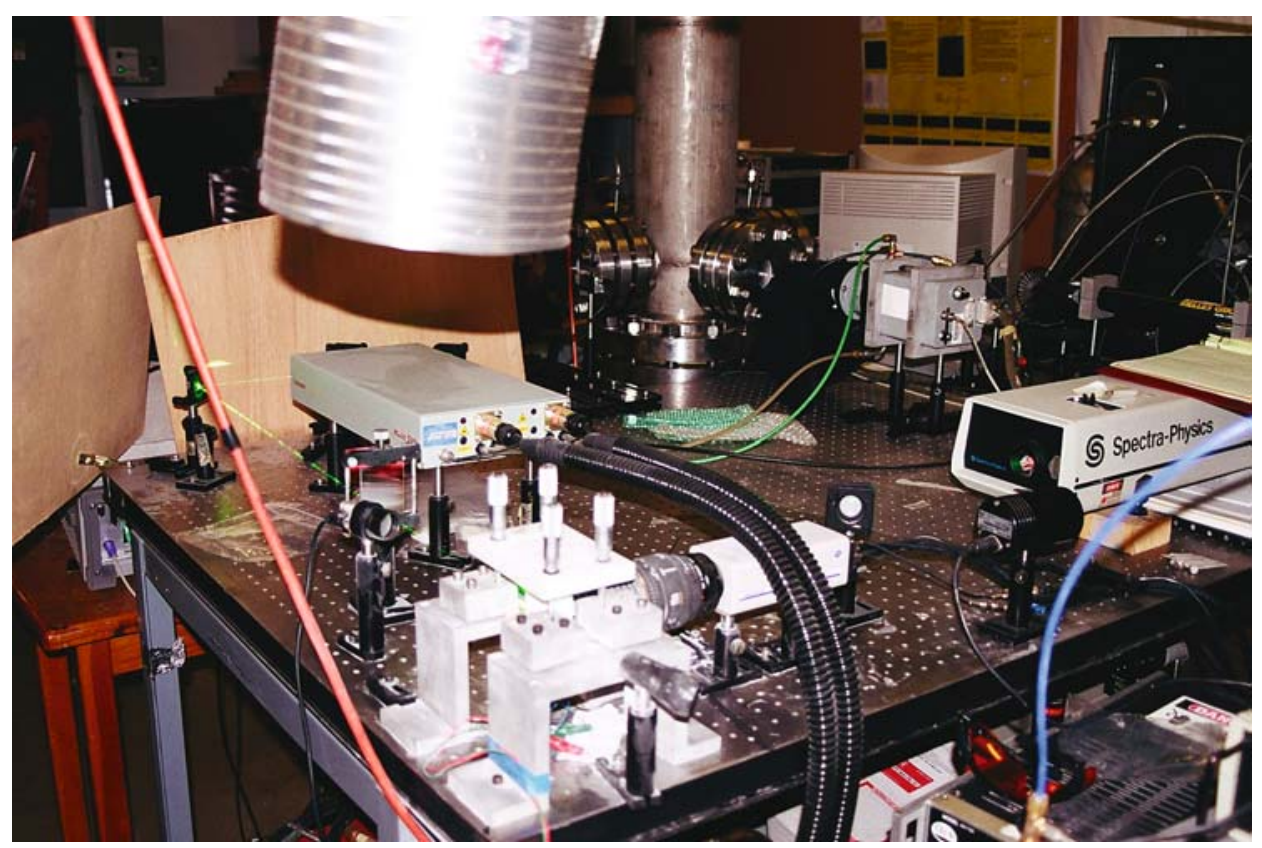

Figure 3. Experimental setup for the air flow visualization.

view. The camera used in the experiment was a digital camera with a $1 \mathrm{k}$ by $1 \mathrm{k}$ chip.

\subsection{EXPERIMENTAL METHODS AND IMPLEMENTATION}

The PTV method relies on the accurate control of exposure time. Extended exposure times allow the camera to capture the movement of the particles in one frame. The particles appear as streaks on the flow trajectories. The exposure time needed to be long enough to allow the seed particles to trace out a streak to describe the trajectories in the induced air flow field. The exposure time was chosen to represent some multiple of the cycle time. This allowed the image to represent a certain number of cycles in the vibration of the piezoelectric bimorph. The image acquisition software used for this experiment allowed accurate and repeatable selection of the exposure time.

\section{Resutls and Discussions}

\subsection{ACOUSTIC STREAMING FIELD AND COOLING EFFECTS}

The acoustic streaming obtained computationally is shown in vector and streamline forms in Figures $4 a$ and $b$. The flow field exhibits combinations of symmetric and counter-rotational eddies, which occupy the whole channel width. The maximum 


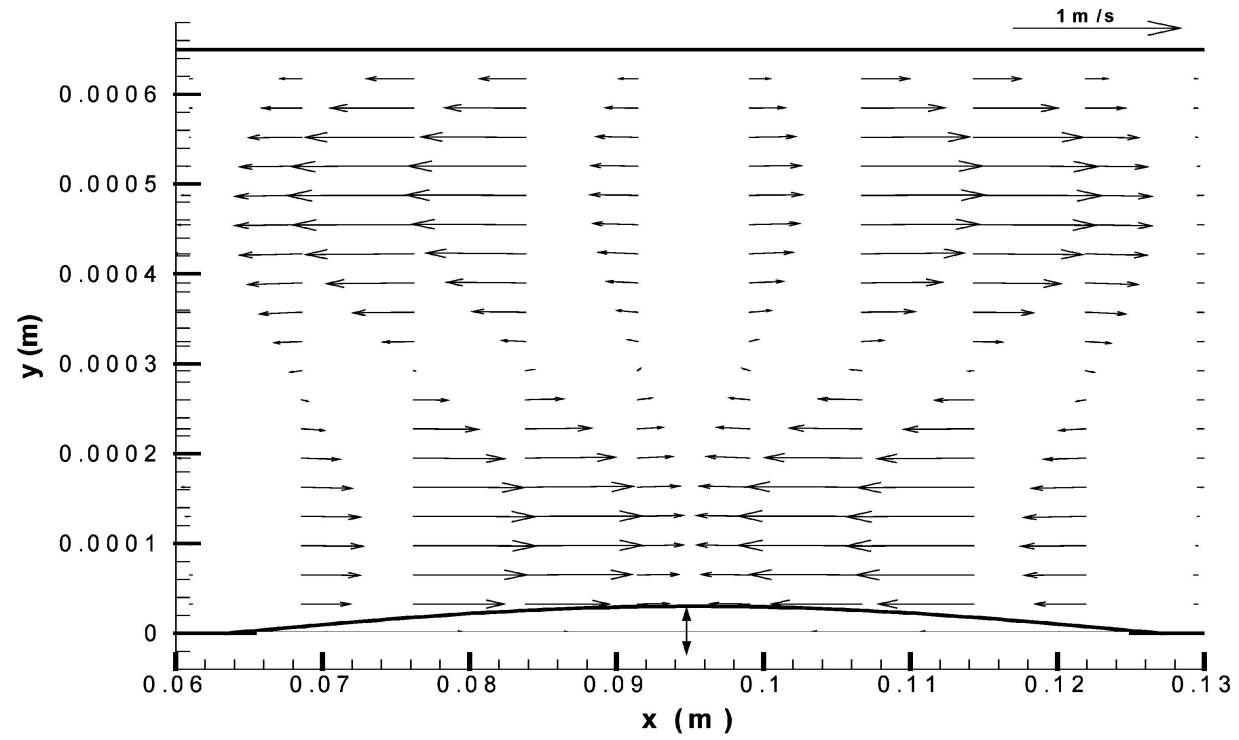

(a) vectors

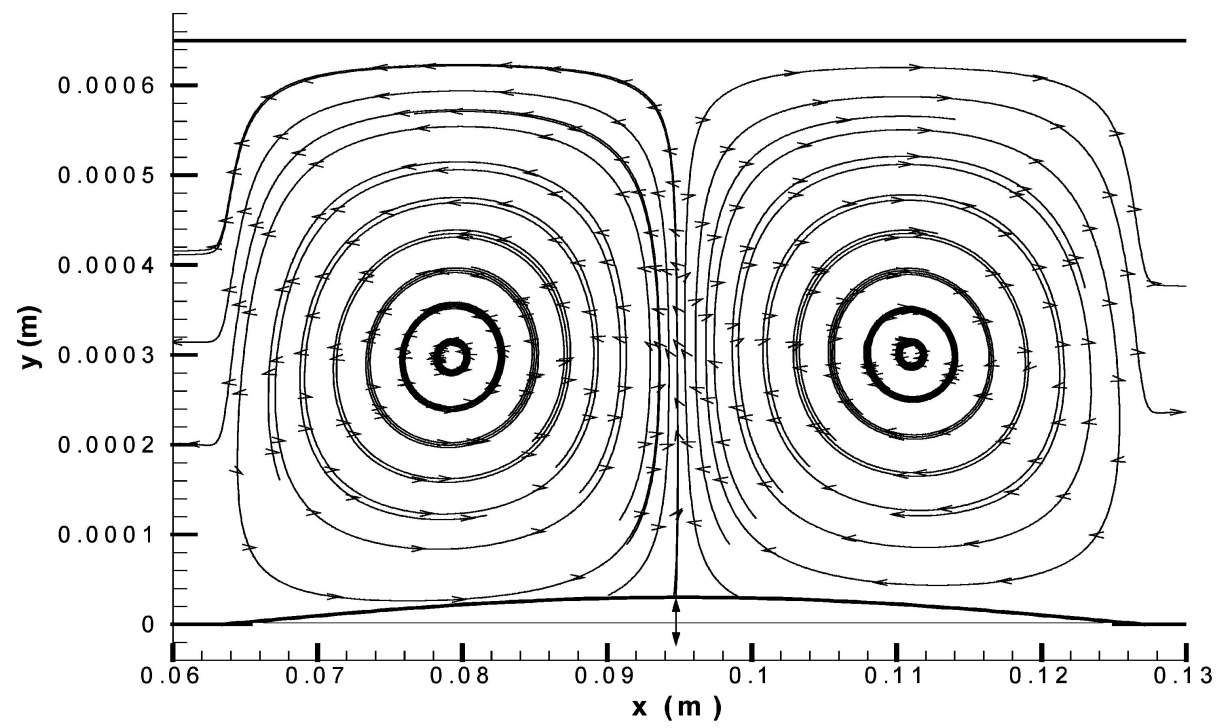

(b) streamlines

Figure 4. Acoustic streaming velocity field $\mathbf{u}_{\mathrm{dc}}$ in the form of vectors (a) and streamlines (b).

streaming velocity in the streaming eddies is found to be $0.821 \mathrm{~m} / \mathrm{s}$ at two symmetric locations, namely, at $(0.08001 \mathrm{~m}, 0.00013 \mathrm{~m})$ and $(0.11049 \mathrm{~m}, 0.00013 \mathrm{~m})$, in the present coordinates. These two locations are horizontally $0.01524 \mathrm{~m}$ away from the anti-node and $0.00013 \mathrm{~m}$ above it. The anti-node is the position on the vibrating beam where the amplitude of the standing wave has its local maximum while the node is the position that does not oscillate (the amplitude equals zero). 
The streaming flow goes from the anti-node, goes down two nodes, and then moves from two nodes to the anti-node along the vibrating beam. This behavior is in agreement with the experimental results obtained in Loh et al. [6] and the numerical results obtained in Wan and Kuznetsov [3].

Temperature fields with and without acoustic streaming are displayed in Figures $5 \mathrm{a}$ and $\mathrm{b}$ respectively. For the case without acoustic streaming (cf. Figure 5a), heat transfer within the channel is dominated by conduction, and the averaged temperature difference $\theta$ along the heat source surface is $102.9^{\circ} \mathrm{C}$. When acoustic streaming is induced in the channel (cf. Figure 5b), $\theta$ drops to $96.8^{\circ} \mathrm{C}$ and more heat is dissipated towards the open end, which is shown by the isothermal lines. Additional cooling effect of the acoustic streaming is a $6.1{ }^{\circ} \mathrm{C}$ or $6 \%$ reduction in temperature compared to the case with no beam vibration. Because of the much larger heat conductivity of the silicon heat source than that of the air in the channel, there is little temperature variation within the heat source, as shown in Figure 6, and along its surface. This is in agreement with Wan and Kuznetsov [3].

Since there are two open ends of the channel, the vibrating acoustic field pushes the fluid in and out of the channel. Because the temperature within the channel is higher than the ambient temperature, the question arises as to whether the acoustic field has any cooling effect (theoretically this field is adiabatic). A rough estimation is made by using a first order acoustic velocity field in an unsteady energy equation and assuming the temperature at two open ends to be ambient. Computations are carried using the meshes described above. The steady-state temperature along the heat source is found to be $93.8^{\circ} \mathrm{C}$, and the temperature drop is $9.1{ }^{\circ} \mathrm{C}$, which means an $8.8 \%$ reduction compared to the case with no beam vibration. These results are listed in Table I. The higher temperature difference for the case with no beam vibration obtained in computations may be due to the 2-D numerical model that does not account for cooling effect in the span-wise dimension, which cannot be eliminated in the experiments. The relative reduction of the temperature drop

Table I. Comparison of the cooling effect of the acoustic streaming obtained experimentally and numerically

\begin{tabular}{|c|c|c|c|c|}
\hline $\begin{array}{l}\text { Temperature } \\
\text { difference from } \\
\text { the ambient } \\
\text { (bimorph), } \theta\left({ }^{\circ} \mathrm{C}\right)\end{array}$ & $\begin{array}{l}\text { Basic } \\
\text { case } \\
\text { (no } \\
\text { vibration) }\end{array}$ & $\begin{array}{l}\text { Vibration } \\
\text { is on }\end{array}$ & $\begin{array}{l}\text { Reduction } \\
\text { of the } \\
\text { temperature } \\
\text { difference }\end{array}$ & $\begin{array}{l}\text { Reduction of the } \\
\text { temperature difference } \\
\text { as the percentage of the } \\
\text { temperature difference } \\
\text { form the basic case }\end{array}$ \\
\hline Experiment & 70.2 & 63.2 & 7 & $9.97 \%$ \\
\hline \multicolumn{5}{|l|}{ Computation } \\
\hline Acoustic streaming cooling & 102.91 & 96.7656 & 6.1444 & $5.97 \%$ \\
\hline Acoustic cooling & 102.91 & 93.84 & 9.07 & $8.82 \%$ \\
\hline Total cooling effect & 102.91 & 87.6956 & 15.2144 & $14.79 \%$ \\
\hline
\end{tabular}

(Ambient air temperature is $20^{\circ} \mathrm{C}$ ) 


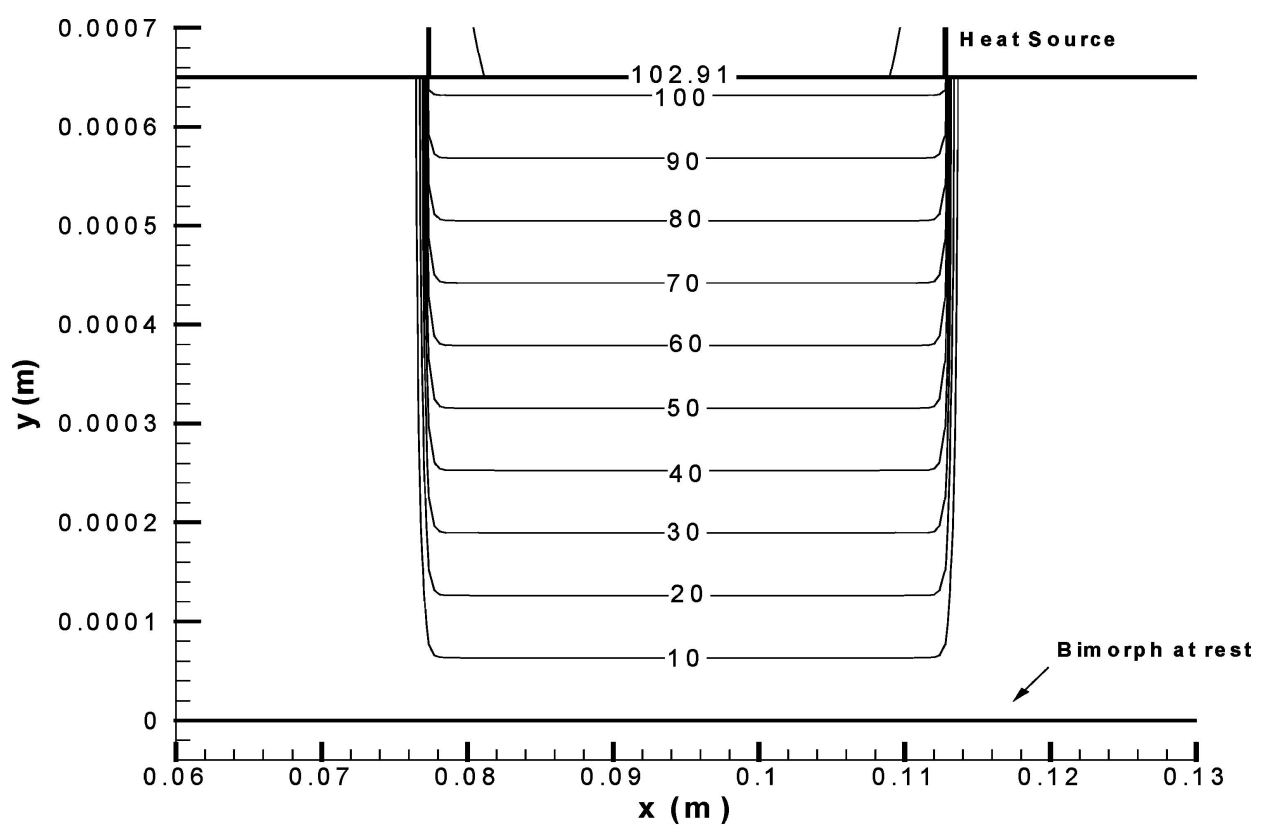

(a) bimorph at rest

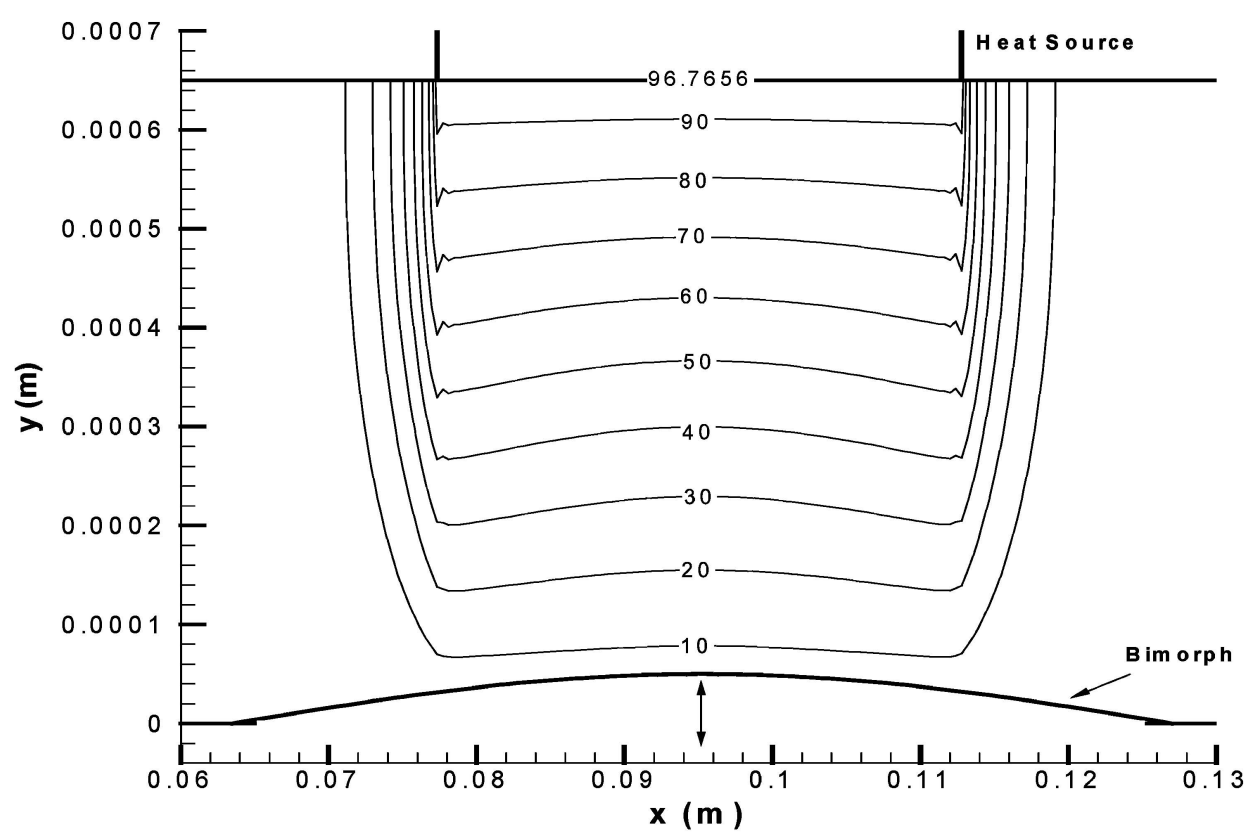

(b) vibrating bimorph

Figure 5. Temperature fields $\theta$ in the gap with bimorph at rest (a) and vibrating (b). 


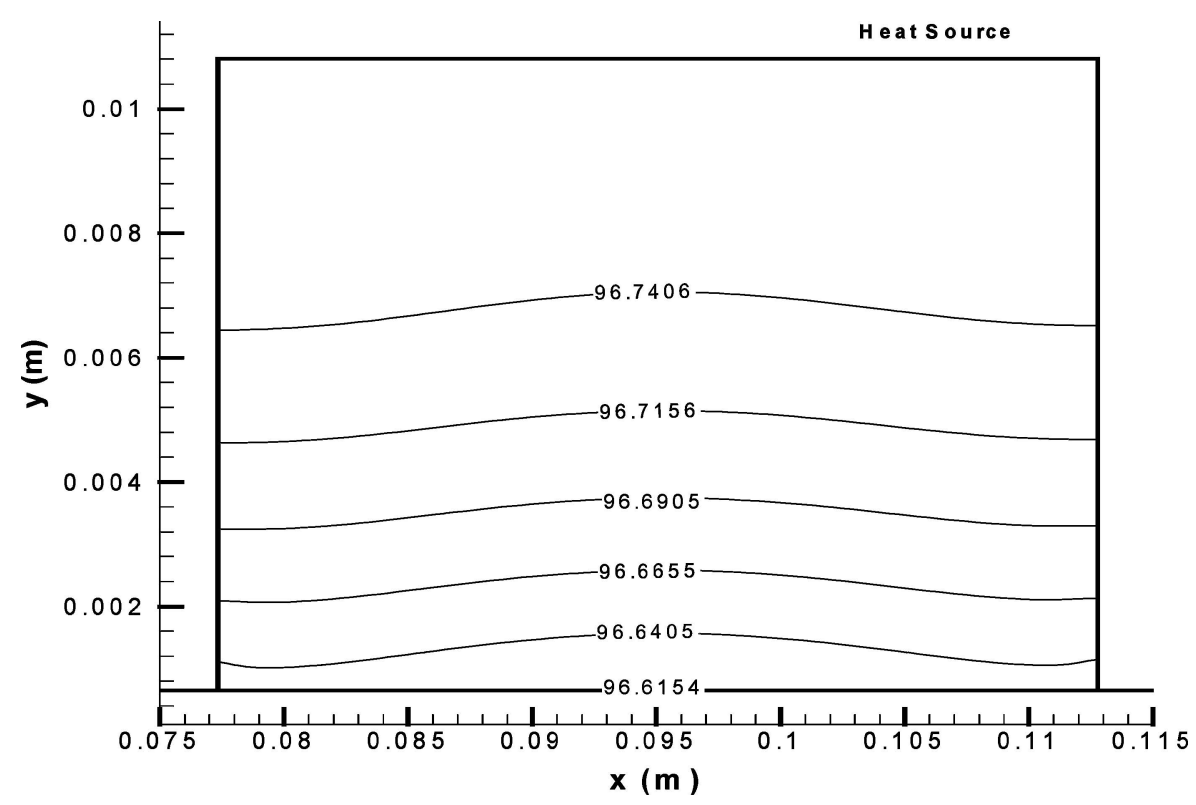

Figure 6. Temperature field $\theta$ in the heat source with vibrating bimorph.

obtained computationally is larger than that obtained experimentally. This may be due to setting the ambient temperature to that of the bimorph in computations. In the experiments, the bimorph temperature is higher than ambient so as the dissipation of the heat from the channel. The comparison of the experimental and numerical results in Table I also reveals that the acoustic streaming is not the only cooling mechanism for the present configuration. The acoustic field may also contribute to the cooling.

\subsection{FLOW PATTERNS GENERATED BY PIEZOELECTRIC BIMORPH VIBRATIONS}

The results for the visualization experiment are images of the air flow field induced by the vibrating piezoelectric bimorph. The flow patterns between the heat source and the vibrating bimorph were analyzed for three different gap sizes. No power was applied to the heat source. For each gap size five images were acquired, which reveals the nature of the flow pattern.

The analysis of flow patterns reveals that the flow field around the bimorph is laminar. The flow around the bimorph did not show any circular flow patterns. The flow did show a clear pattern where the particle streaks originated in the center and flowed upward away from the bimorph and toward the outside of the bimorph.

Figure 7 describes the flow surrounding the piezoelectric bimorph. The gap dimension was three millimeters, and the exposure time was $321 \mathrm{~ms}$, corresponding 


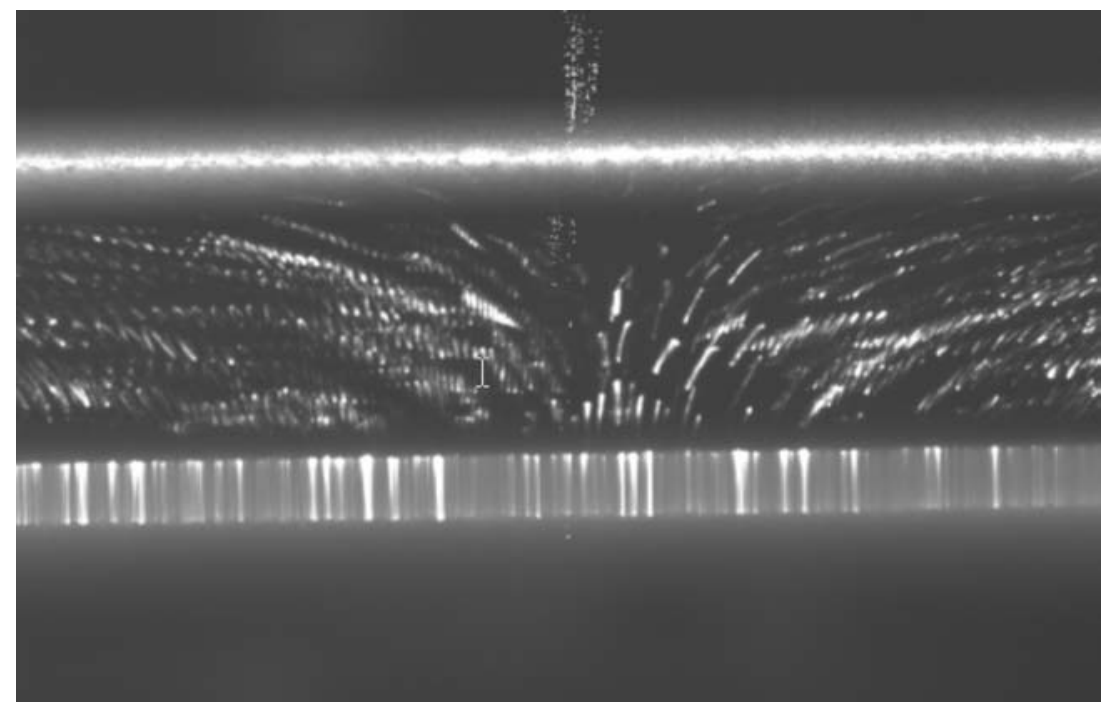

Figure 7. Flow pattern for non-slot bimorph $(\mathrm{Gap}=3 \mathrm{~mm})$.

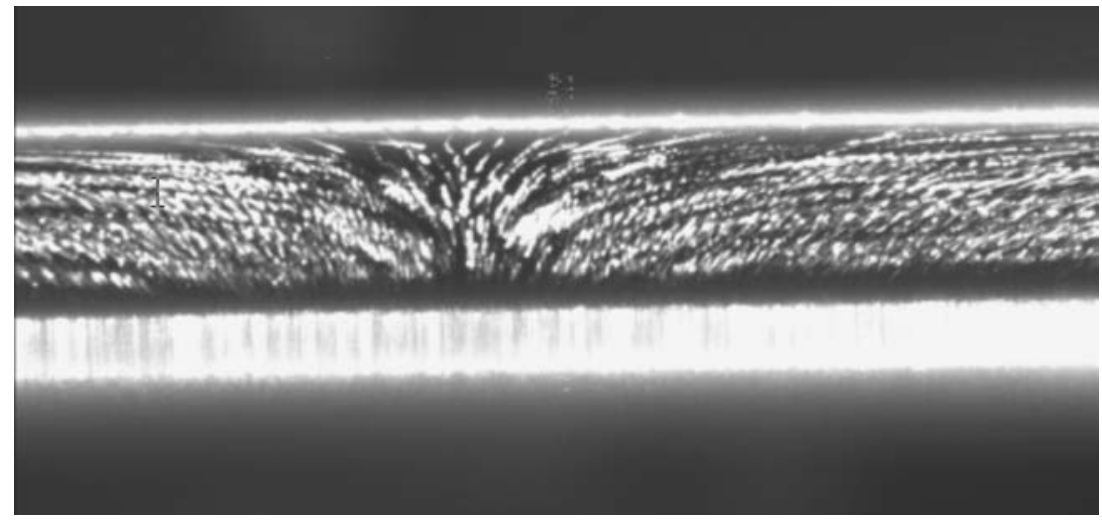

Figure 8. Flow pattern for non-slot bimorph $(\mathrm{Gap}=2 \mathrm{~mm})$.

to 90 bimorph oscillation cycles. It is interesting to note the vertical trajectories in the center of the bimorph. The trajectories towards the sides of the bimorph tend to flow upward and away from the center.

Figure 8 shows the same descriptive flow as in Figure 7. This image was taken with the same exposure time of $321 \mathrm{~ms}$, but the gap was reduced to $2 \mathrm{~mm}$. In this case the flow is primarily horizontal. The flow in the direct center of the bimorph flows vertically. Flow across the upper plate would have a greater impact on the convective heat transfer. When the gap size was further reduced to one millimeter, the analysis of the flow in the gap was difficult to perform due to the small gap (see Figure 9). 


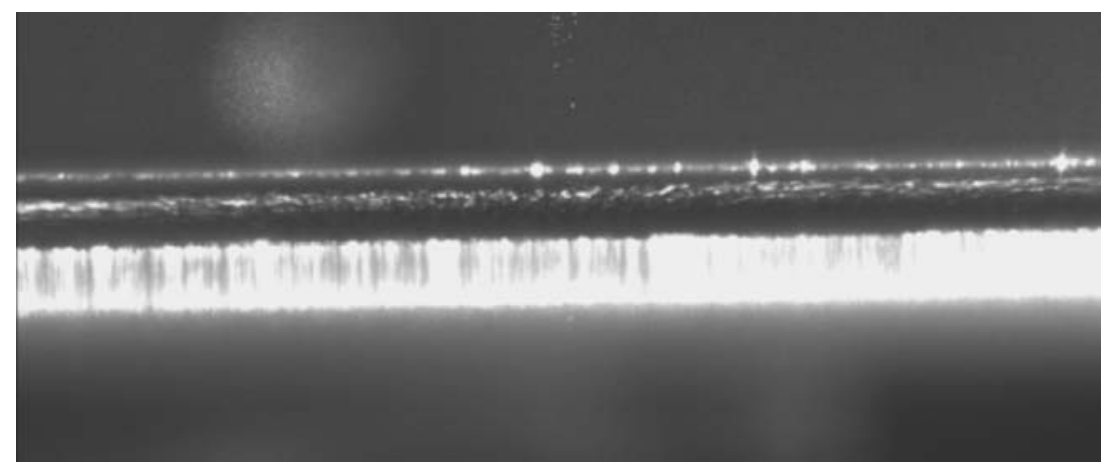

Figure 9. Flow pattern for non-slot bimorph $(\mathrm{Gap}=1 \mathrm{~mm})$.

In summary, the above visualization clearly shows that a vertical streaming (acoustic streaming) can be induced by bimorph vibration, which may be responsible for the enhancement of the heat transfer between the heat source and the surrounding air.

\section{Conclusions}

The combined experimental and numerical study presented in this paper indicates a potential of acoustic streaming for different cooling applications, such as removal of dissipated heat from integrated circuit boards. The air flow visualization by PTV shows that the acoustic streaming can be induced by the bimorph vibration, which may be responsible for the calculated and measured enhancement of the heat transfer between the heat source and the surrounding air. Numerical model for the cooling efficiency of the bimorph is also established. The comparisons of the computational results with the experimental results show that the acoustic streaming has a definite cooling effect. Further research is needed before a particular cooling device based on this principal is developed.

\section{References}

1. Bradley, C.E., Acoustic streaming field structure: The influence of radiator. J. Acoust. Soc. Am. 100 (1996) 1399-1408.

2. Nyborg, W.L., Acoustic streaming. in Mason, W.P. (ed.), Physical Acoustics, Vol. 2B, Academic Press, New York (1965) pp. 265-331.

3. Wan, Q. and Kuznetsov, A.V., Numerical study of the efficiency of acoustic streaming for enhancing heat transfer between two parallel beams. Flow Turbul. Combust. 70 (2003) 89-114.

4. Givoli, D., Non-reflecting boundary conditions. J. Comp. Phys. 94 (1991) 1-29.

5. Wan, Q. and Kuznetsov, A.V., Streaming in a channel bounded by an ultrasonically oscillating beam and its cooling efficiency. Numer. Heat Trans. A 45 (2004) 21-47.

6. Loh, B.G., Hyun, S., Ro, P.I. and Kleinstreuer, C., Acoustic streaming induced by ultrasonic flexural vibrations and associated enhancement of convective heat transfer. J. Acoust. Soc. Am. 111 (2002) 875-883. 